Artigo original

\title{
Comparação entre os valores obtidos e previstos da força muscular respiratória em uma coorte de adultos saudáveis da região oeste de São Paulo Comparison between the obtained and predicted values of the respiratory muscle strength in a cohort of healthy adults of the São Paulo west region
}

Thaís de Lima Laranjeira*, Maria Imaculada de Lima Montebelo*, Daniela Ike**, Karina Maria Cancelliero**, Dirceu Costa***

*Programa de Pós-graduação e Curso de Graduação em Fisioterapia da UNIMEP, Piracicaba/SP, **Programa de Pós-graduação em Fisioterapia da UFSCAR, ***Professor do Programa de Pós-graduação em Fisioterapia da UFSCAR e Ciências da Reabilitação da UNINOVE

\section{Resumo}

Objetivo: Avaliar a força muscular respiratória pela pressão inspiratória máxima (PImáx) e pressão expiratória máxima (PEmáx), verificando a correlação entre os valores obtidos e previstos pela equação de Neder $e$ t al. em indivíduos saudáveis. Método: Foi realizada uma avaliação da força muscular respiratória em 128 indivíduos saudáveis (ambos os sexos) com um manovacuômetro analógico. Para o cálculo dos valores previstos da PImáx e PEmáx foram utilizadas as equaçôes propostas por Neder $e t a l$. Para a análise estatística foram utilizados o teste Shapiro-Wilk e o teste de correlação de Spearman. Resultados: A análise de correlação foi realizada entre os valores obtidos de PImáx e PEmáx de homens e mulheres com os valores propostos pela equação de Neder et al. Os valores de PImáx, tanto para os homens quanto para as mulheres, foram inferiores aos valores preditos, exceto na faixa etária de 61-70 anos do sexo feminino. Os valores da PEmáx, em ambos os sexos, foram, em sua maioria, inferiores aos valores da equação apresentando apenas um resultado significativo para a faixa etária de 31-40 anos do sexo masculino. Conclusão: Os valores obtidos no nosso estudo foram inferiores aos previstos pela equação de Neder $e t a l$. indicando a necessidade de novos estudos para contribuir na equaçáo de valores previstos para a PImáx e PEmáx, em indivíduos da população brasileira.

Palavras-chave: força muscular, fisioterapia, músculos respiratórios.

\begin{abstract}
Objective: To evaluate the respiratory muscle strength by maximal inspiratory pressure (MIP) and the maximal expiratory pressure (MEP), observing the correlation between obtained and predicted values by Neder $e$ al . equation in healthy subjects. Methods: It was carried out an evaluation of respiratory muscle strength in 128 healthy subjects (both gender) with an aneroid manometer/vacuometer. For the calculation of MIP and MEP predicted values, the equation proposed by Neder et al. was used. For the statistical analysis the Shapiro-Wilk test and the Spearman correlation test were used. Results: The correlation analysis was performed between MIP and MEP obtained values of men and women with the proposed values by the Neder et al. equation. Both for men and women the MIP values were inferior to the predicted values, except for the female gender in the 61-70 age group. The MEP values, in both genders, were inferior to the equation values, showing only one significant result for the male gender in the 31-40 age group. Conclusion: The obtained values in our study were inferior to the predicted values of Neder $e t a l$. and we concluded that more studies may contribute to the equation of predicted values for Brazilian population individuals.
\end{abstract}

Key-words: muscle strength, physical therapy, respiratory muscles. 


\section{Introdução}

A força muscular respiratória é definida como sendo a máxima e a mínima pressão mensurada ao nível da boca, devido a um esforço muscular necessário à mudança de pressão [1,2]. Ela pode ser mensurada por meio das pressóes sub e supra-atmosférica que esses músculos são capazes de gerar, sendo, portanto, a pressão inspiratória máxima (PImáx) a maior pressão que pode ser gerada durante a inspiração forçada contra uma via aérea ocluída; e a pressão expiratória máxima (PEmáx), a maior pressão que pode ser desenvolvida durante um vigoroso esforço expiratório contra uma via aérea ocluída [3].

A avaliação da força muscular respiratória a partir das pressóes respiratórias máximas tem sido amplamente estudada nas últimas décadas. A medida quantitativa da função dos músculos respiratórios mais simples e amplamente utilizada é a pressão respiratória estática máxima gerada na boca [4]. A mensuração dessas pressóes respiratórias máximas é um procedimento simples, rápido e não-invasivo.

Em 1969, Black e Hyatt [5] introduziram uma forma simples de mensuração das pressões respiratórias máximas por meio de um manovacuômetro em escala de $\mathrm{cmH}_{2} \mathrm{O}$ (centímetros de água). Essa escala também pode ser em mmHg (milímetros de mercúrio) [6] e pelo uso de uma coluna d'água $[7,8]$. Contudo, é importante considerar que este método avalia somente a força do conjunto dos músculos inspiratórios ou expiratórios, mas não de cada músculo isoladamente [9].

De acordo com Mangelsdorff et al. [10], a PImáx representa a força desenvolvida pelos músculos inspiratórios. Com relação à PEmáx, esta é uma medida que indica a força dos músculos abdominais, intercostais internos e do triângulo esternal $[11,12]$. Essas medidas são importantes na avaliação da força dos músculos respiratórios em diferentes condiçôes como em indivíduos saudáveis de diferentes faixas etárias; em pacientes com distúrbios de diferentes origens; no pré e pósoperatório de cirurgias tóracoabdominais, e em treinamento muscular respiratório $[3,13,14]$.

Frente à importância da mensuração das pressões respiratórias máximas, muitos estudos surgiram na tentativa de se estabelecerem tabelas de valores de referências, considerandose fatores como idade, sexo e altura, em diferentes populações [5,14-19]. Black e Hyatt [5] realizaram um estudo com 120 indivíduos saudáveis, de ambos os sexos, com idade entre 20 e 86 anos, determinando os valores de pressóes respiratórias máximas e equações de referência para a população saudável, levando em consideração sexo e idade. Wilson et al. [17] mediram a PImáx e PEmáx de 370 adultos e crianças caucasianos britânicos, relacionando idade, altura e peso para formular equaçóes de valores previstos, e constataram que nos homens tanto a PImáx quanto a PEmáx se correlacionaram significativamente apenas com a idade, enquanto que nas mulheres houve correlação com a altura. Bruschi et al. [18] realizaram um estudo para definir valores de referência das pressões respiratórias máximas em 625 indivíduos saudáveis (266 homens e 359 mulheres) na população italiana, tendo como parâmetros a idade, o sexo e a área de superfície corporal. Já Johan et al. [19], em 1997, fizeram um estudo comparando os valores das pressóes (PImáx e PEmáx) em adultos chineses, malásios e indianos, verificando diferenças étnicas significativas nas pressóes respiratórias.

No Brasil, Camelo et al. [20] foram os primeiros a descrever valores de PImáx e PEmáx, avaliando 60 indivíduos saudáveis de ambos os sexos, com idade entre 20 e 49 anos. Neder et al. [3] propuseram fórmulas de valores previstos para homens e mulheres, em diferentes idades. Parreira et al. [13] recrutaram 47 homens e 56 mulheres saudáveis, não fumantes, com idade entre 20 a 80 anos, com a finalidade de comparar os valores encontrados das pressóes respiratórias máximas em uma amostra de indivíduos saudáveis de Minas Gerais com valores preditos pelas equaçóes propostas por Neder et al. [3].

Contudo, as equaçóes para tais valores previstos, ao serem colocadas na prática clínica, nem sempre se correlacionam com os valores obtidos, justificando a necessidade de novos estudos sobre este tema, principalmente pela sua importância e uso frequente na fisioterapia respiratória.

\section{Objetivos}

O objetivo deste trabalho foi avaliar a força muscular respiratória, representada pela pressão inspiratória máxima (PImáx) e a pressão expiratória máxima (PEmáx), verificando a correlação entre os valores obtidos e previstos pela equação de Neder et al. [3], em ambos os sexos, abrangendo uma faixa etária de 20 a 80 anos em indivíduos saudáveis na população brasileira, especificamente na região de Piracicaba/SP.

\section{Material e métodos}

\section{Casuística}

Participaram deste estudo, 128 indivíduos saudáveis de 20 a 80 anos, sendo 62 homens e 66 mulheres, que não apresentavam histórico de doenças pulmonares e hipertensão, os quais foram selecionados aleatoriamente, provenientes de diversas cidades na regiáo de Piracicaba/SP. Foram excluídos os pacientes com doenças pulmonares, cardiovasculares, neurológicas e disfunçôes ortopédicas que impedissem a realização dos testes. Os procedimentos do estudo foram aprovados pelo Comitê de Ética da Instituição (Processo n. 07/2007) e todos os indivíduos assinaram o Termo de Consentimento Livre e Esclarecido. Além disso, semelhante ao estudo de Neder et al., os indivíduos foram estratificados em subgrupos de acordo com a idade (20-30, 31-40, 41-50, 51-60, 61-70, 71-80 anos) e gênero, apresentando um total de 12 estratos. 


\section{Procedimento inicial}

Inicialmente, foi realizada a anamnese, com o preenchimento de uma ficha de avaliação, constituída de questóes referentes ao tabagismo, prática de atividade física regular, presença de disfunçóes cardiovasculares e respiratórias, antecedentes familiares e medicamentos em uso. Após esse procedimento, foi realizada a aferição da pressão arterial, por meio de um esfignomanômetro da marca Tycos ${ }^{\circledR}$, e avaliação antropométrica mensurando o peso e a altura do indivíduo por meio de uma balança antropométrica manual da marca Welmy ${ }^{\circ}$ de 0 a $150 \mathrm{~kg}$, sendo que, posteriormente foi calculado o índice de massa corporal (IMC), pela fórmula peso/ altura $^{2}$

Com relação ao período das coletas, essas foram realizadas no período vespertino com todos os voluntários.

\section{Avaliação da força muscular respiratória}

Para a mensuração da força muscular respiratória (PImáx e PEmáx) foi utilizado um manovacuômetro analógico escalonado em $\mathrm{cmH}_{2} \mathrm{O}$ com variação de +300 a -300 $\mathrm{cmH}_{2} \mathrm{O}$ da marca Ger-Ar Sáo Paulo, Brasil. O mesmo foi conectado a uma traquéia com $1 \mathrm{~m}$ de comprimento e $2 \mathrm{~cm}$ de diâmetro interno, sendo que sua extremidade foi conectada a um bocal, contendo um orifício de aproximadamente $2 \mathrm{~mm}$ de diâmetro, com a finalidade de proporcionar um escape de ar e, assim, prevenir a elevação da pressão da cavidade oral gerada, exclusivamente, pela contração indesejada dos músculos da parede bucal, evitando interferência destes nos resultados, conforme recomendaçôes de Camelo et al. [20].

O aparelho foi calibrado anteriormente ao início do estudo, segundo recomendaçóes do Inmetro, por meio da aplicação crescente de pressão e vácuo até o limite estabelecido pelo fabricante do equipamento. Anteriormente a cada teste, foi realizada uma verificação da posição do ponteiro no ponto zero e, caso necessário, um simples ajuste do parafuso do ponteiro foi realizado.

As manobras para a medida da força muscular respiratória foram realizadas com o voluntário na posição sentada, estando o tronco num ângulo de $90^{\circ}$ com o quadril e com um clipe nasal colocado em toda manobra. A PImáx foi mensurada a partir do Volume Residual (VR), ou seja, após uma expiração máxima, enquanto a PEmáx foi mensurada a partir da capacidade pulmonar total (CPT), após o indivíduo realizar uma inspiração máxima [21]. Todos os indivíduos realizaram no mínimo três medidas reprodutíveis, sendo sustentadas por dois segundos [3]. Com relação ao intervalo entre as três manobras, o voluntário fazia a próxima de acordo com o descanso necessário individual para realizá-la, o que durou aproximadamente três minutos.

Todos os indivíduos foram avaliados por apenas um examinador durante todos os procedimentos executados.
Neste estudo, os valores de PImáx e PEmáx encontrados foram comparados com os valores preditos pelas equaçóes propostas por Neder et al. a saber:

Homens: PImáx: $y=-0,80$ (idade) $+155,3$; SEE $=17.3$ PEmáx: $y=-0,81$ (idade) $+165,3$; SEE $=15.6$

Mulheres: PImáx: $y=-0,49$ (idade) $+110,4 ;$ SEE $=9.1$ PEmáx: $y=-0,61$ (idade) $+115,6 ;$ SEE $=11.2$

\section{Análise dos dados}

Para análise dos dados, foi, inicialmente, computado o maior valor das pressóes máximas, desde que este não excedesse $10 \%$ em relação ao valor mais próximo. No tratamento estatístico dos dados, aplicou-se o teste de normalidade de Shapiro-Wilk e, para verificar a associação entre esses valores previstos e observados, foi utilizado o coeficiente de correlação de Spearman, sendo considerados como diferença significativa valores de $\mathrm{p}<0,05$. Utilizando o programa Statistical Package for the Social Sciences (SPSS) na versão 13.0, sendo que os valores estão expressos em média \pm desvio-padrão.

Para a avaliaçáo da concordância entre dois métodos de medida, foi utilizado o teste e o gráfico de Bland-Altman, pelo programa Medcalc.

\section{Resultados}

Primeiramente, pode-se observar na Tabela I os dados antropométricos e demográficos da amostra, bem como a distribuição dos indivíduos em relação ao gênero e a faixa etária, ressaltando que foram avaliados 128 indivíduos (62 homens e 66 mulheres).

Os dados foram apresentados separadamente para mulheres e homens. Quanto à PImáx, pode-se observar na Tabela II, que os valores obtidos, tanto nos homens quanto nas mulheres, foram inferiores aos valores preditos, exceto na faixa etária de 61-70 anos nas mulheres $(\mathrm{p}<0,05)$, no qual houve valor significante de correlação.

Com relação aos valores da PEmáx, tanto para os homens quanto para as mulheres, foram, em sua maioria, inferiores aos valores preditos pela equação de Neder $e t a l$. [3] conforme está descrito na Tabela III, apresentando apenas um resultado significante de correlação na faixa etária de 31-40 anos nos homens $(\mathrm{p}<0,05)$.

O coeficiente de correlaçáo intraclasse (ICC) mostrou concordância baixa para todas as dimensóes, sendo que os valores se apresentaram abaixo de 0,40 ; exceto para os valores de PImáx do sexo masculino, que apresentou um ICC médio, pois o índice foi igual a 0,53 , como mostra a Tabela IV.

As Figuras 1, 2, 3 e 4 representam os gráficos de BlandAltman de concordância entre os valores de PImáx e PEmáx obtidos e previstos, mostrando que houve concordância adequada entre os valores previstos comparados com os obtidos pela fórmula de Neder et al. [3]. 
Tabela I - Características antropométricas e demográficas subdivididas em gênero e faixa etária.

\begin{tabular}{|c|c|c|c|c|c|c|}
\hline & $\begin{array}{c}\text { Faixa etária } \\
\text { (anos) }\end{array}$ & $\begin{array}{l}\text { Média de idade } \\
\text { (anos e meses) }\end{array}$ & Peso $(\mathrm{kg})$ & $\begin{array}{l}\text { Estatura } \\
\text { (metros) }\end{array}$ & $\mathrm{IMC}\left(\mathrm{kg} / \mathrm{m}^{2}\right)$ & $\begin{array}{l}\text { Distribuição por } \\
\text { faixa etária (n) }\end{array}$ \\
\hline \multicolumn{7}{|l|}{ Mulheres } \\
\hline & $20-30$ & $24,31 \pm 2,98$ & $66,40 \pm 17,90$ & $1,62 \pm 0,06$ & $25,32 \pm 6,10$ & 13 \\
\hline & $31-40$ & $35,90 \pm 2,73$ & $66,95 \pm 17,73$ & $1,63 \pm 0,09$ & $25,68 \pm 4,38$ & 10 \\
\hline & $41-50$ & $45,50 \pm 3,27$ & $71,85 \pm 10,28$ & $1,62 \pm 0,03$ & $27,32 \pm 3,86$ & 10 \\
\hline & $51-60$ & $56,20 \pm 2,78$ & $73,07 \pm 11,48$ & $1,62 \pm 0,10$ & $28,80 \pm 3,00$ & 10 \\
\hline & $61-70$ & $65,40 \pm 3,14$ & $75,05 \pm 12,81$ & $1,59 \pm 0,06$ & $29,52 \pm 4,38$ & 15 \\
\hline & $71-80$ & $75,56 \pm 3,47$ & $62,89 \pm 10,70$ & $1,57 \pm 0,07$ & $25,69 \pm 4,84$ & 09 \\
\hline \multicolumn{7}{|l|}{ Homens } \\
\hline & $20-30$ & $25,69 \pm 2,69$ & $82,77 \pm 20,15$ & $1,78 \pm 0,09$ & $23,69 \pm 2,51$ & 13 \\
\hline & $31-40$ & $34,80 \pm 3,43$ & $93,54 \pm 13,00$ & $1,79 \pm 0,06$ & $31,27 \pm 4,74$ & 10 \\
\hline & $41-50$ & $46,22 \pm 2,54$ & $84,99 \pm 7,53$ & $1,75 \pm 0,05$ & $27,81 \pm 3,33$ & 09 \\
\hline & $51-60$ & $55,20 \pm 2,74$ & $77,45 \pm 10,11$ & $1,71 \pm 0,07$ & $26,73 \pm 2,93$ & 10 \\
\hline & $61-70$ & $65,63 \pm 2,77$ & $74,75 \pm 9,32$ & $1,70 \pm 0,05$ & $25,87 \pm 2,23$ & 10 \\
\hline & $71-80$ & $76,20 \pm 3,63$ & $72,20 \pm 6,81$ & $1,67 \pm 0,05$ & $25,73 \pm 1,43$ & 10 \\
\hline
\end{tabular}

Valores expressos em média \pm desvio padrão.

Tabela II - Correlação entre os valores preditos e observados da PImáx sub-divididas em gênero e faixa etária.

\begin{tabular}{llllll}
\hline \multicolumn{1}{c}{ Faixa etária } & $\mathbf{n}$ & PImáx (Obtido) & PImáx (Previsto) & $\mathbf{r}_{\mathbf{s}}$ & P valor \\
\hline Mulheres & & & $-98,49 \pm 1,46$ & 0,32 & 0,28 \\
\hline $20-30$ & 13 & $-61,41 \pm 15,50$ & $-92,64 \pm 1,13$ & $-0,07$ & 0,85 \\
$31-40$ & 10 & $-53,33 \pm 9,08$ & $-88,11 \pm 1,60$ & 0,05 & 0,88 \\
$41-50$ & 10 & $-67,33 \pm 19,66$ & $-82,69 \pm 1,32$ & 0,34 & 0,33 \\
$51-60$ & 10 & $-58,33 \pm 19,29$ & $-78,35 \pm 1,54$ & $0,61^{*}$ & $0,02^{*}$ \\
$61-70$ & 15 & $-57,00 \pm 23,35$ & $-73,38 \pm 1,70$ & $-0,004$ & 0,98 \\
$71-80$ & 09 & $-48,33 \pm 18,93$ & & & 0,70 \\
\hline Homens & & & $-134,63 \pm 2,65$ & $-0,12$ & 0,08 \\
\hline $20-30$ & 13 & $-105,83 \pm 35,11$ & $-125,70 \pm 2,83$ & 0,58 & 0,38 \\
$31-40$ & 10 & $-91,11 \pm 23,36$ & $-118,32 \pm 2,03$ & 0,32 & 0,19 \\
$41-50$ & 09 & $-103,15 \pm 36,27$ & $-110,70 \pm 2,21$ & 0,45 & 0,29 \\
$51-60$ & 10 & $-82,92 \pm 21,11$ & $-102,80 \pm 2,22$ & 0,37 & 0,29 \\
$61-70$ & 10 & $-87,08 \pm 21,49$ & $-94,34 \pm 2,91$ & 0,36 & \\
$71-80$ & 10 & $-60,66 \pm 26,81$ & & & \\
\hline
\end{tabular}

Valores expressos em média \pm desvio padrão.

${ }^{*} p<0,05$ pela Correlação de Spearman $\left(r_{s}\right)$

Tabela III - Correlação entre os valores preditos e observados da PEmáx sub-divididas em gênero e faixa etária.

\begin{tabular}{lccccc}
\hline Faixa etária (anos) & $\mathbf{n}$ & PEmáx (Obtido) & PEmáx (Previsto) & $\mathbf{r}_{\mathbf{s}}$ & $\mathbf{P}$ \\
\hline Mulheres & & & & & 0,08 \\
\hline $20-30$ & 13 & $78,08 \pm 13,54$ & $100,77 \pm 1,82$ & $-0,03$ & 0,95 \\
$31-40$ & 10 & $77,14 \pm 13,90$ & $93,49 \pm 1,41$ & 0,12 & 0,73 \\
$41-50$ & 10 & $99,33 \pm 27,16$ & $87,85 \pm 2,00$ & 0,55 & 0,10 \\
$51-60$ & 10 & $83,33 \pm 25,77$ & $81,10 \pm 1,65$ & 0,31 & 0,25 \\
$61-70$ & 15 & $80,22 \pm 24,03$ & $75,71 \pm 1,91$ & $-0,24$ & 0,52 \\
$71-80$ & 09 & $61,30 \pm 14,52$ & $69,51 \pm 2,12$ & $-0,15$ & 0,62 \\
\hline Homens & & & & $0,67^{*}$ & $0,03^{*}$ \\
\hline $20-30$ & 13 & $136,67 \pm 56,96$ & $144,38 \pm 2,68$ & 0,36 & 0,34 \\
$31-40$ & 10 & $127,22 \pm 27,46$ & $135,33 \pm 2,86$ & $-0,04$ & 0,92 \\
$41-50$ & 09 & $131,11 \pm 37,42$ & $127,86 \pm 2,06$ & $-0,13$ & 0,73 \\
$51-60$ & 10 & $131,04 \pm 36,70$ & $120,14 \pm 2,24$ & $-0,47$ & 0,17 \\
$71-70$ & 10 & $130,00 \pm 21,68$ & $112,14 \pm 2,25$ & $103,58 \pm 2,94$ &
\end{tabular}

Valores expressos em média \pm desvio padrão.

${ }^{*} p<0,05$ pela Correlação de Spearman $\left(r_{s}\right)$ 
Tabela IV - Valores e concordância entre as pressóes inspiratória máxima (PImáx) e expiratória máxima (PEmáx) obtidas e previstas dos grupos masculino e feminino.

\begin{tabular}{lccccc}
\hline Pressões respiratórias máximas & $\mathbf{n}$ & Valores obtidos & Valores previstos & ICC* & Valor $\mathbf{p}$ \\
\hline PImáx $\left(\mathrm{cmH}_{2} \mathrm{O}\right)$ & & & & & \\
\hline Feminino & 67 & $-59,33 \pm 19,11$ & $-85,88 \pm 8,77$ & 0,31 & 0,07 \\
Masculino & 61 & $-90,03 \pm 30,30$ & $-115,87 \pm 14,51$ & 0,53 & 0,00 \\
\hline PEmáx $\left(\mathrm{cmH}_{2} \mathrm{O}\right)$ & & & & & \\
\hline Feminino & 67 & $81,52 \pm 22,73$ & $85,07 \pm 10,92$ & 0,27 & 0,099 \\
Masculino & 61 & $122,80 \pm 34,93$ & $125,37 \pm 14,69$ & 0,39 & 0,026 \\
\hline
\end{tabular}

Valores expressos em média \pm desvio padrão.

ICC*: Concordância intraclasse.

Figura 1 - Grau de concordância entre a PImax obtida e a PImax prevista para o grupo masculino, de acordo com o teste de BlandAltman. $\pm 1,96 S D$ : intervalo de confiança de 95\%. Mean (cm): Média da diferença entre as médias.

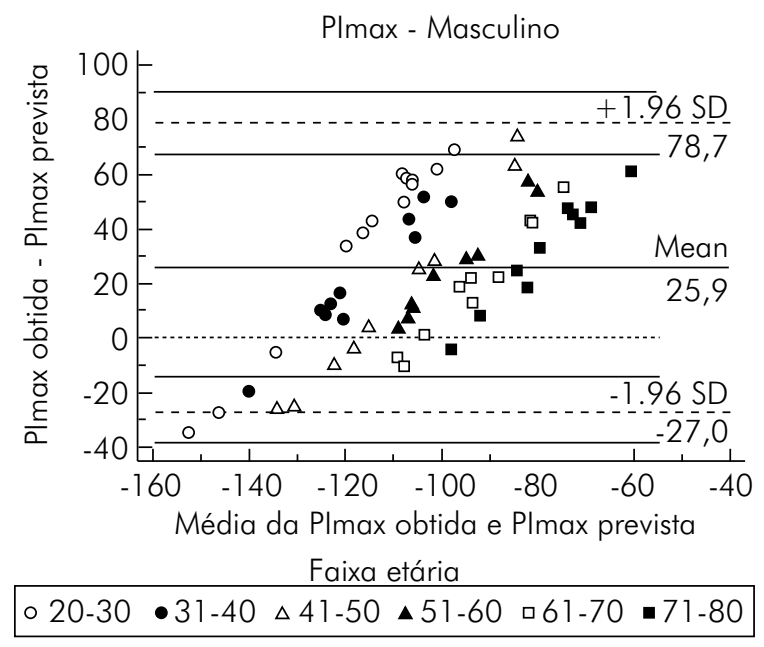

Figura 2 - Grau de concordância entre a PEmax obtida e a PEmax prevista para o grupo masculino, de acordo com o teste de BlandAltman. $\pm 1,965 D$ : intervalo de confiança de 95\%. Mean (cm): Média da diferença entre as médias.

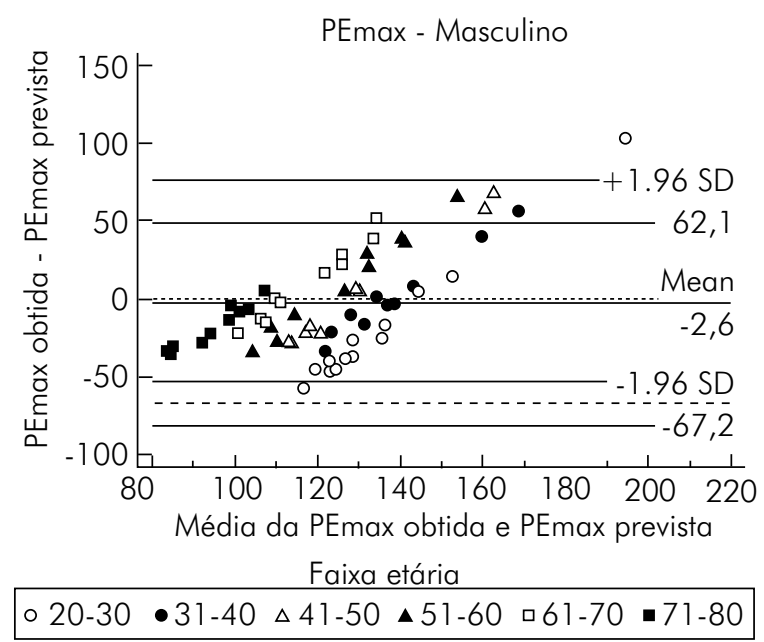

Figura 3 - Grau de concordância entre a PImax obtida e a PImax prevista para o grupo feminino, de acordo com o teste de BlandAltman. $\pm 1,96 S D$ : intervalo de confiança de 95\%. Mean (cm): Média da diferença entre as médias.

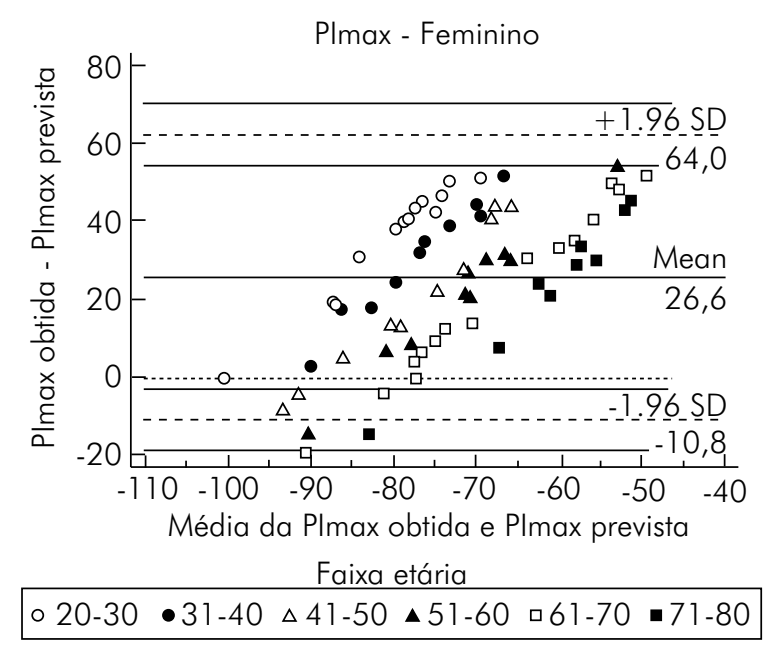

Figura 4 - Grau de concordância entre a PEmax obtida e a PEmax prevista para o grupo feminino, de acordo com o teste de BlandAltman. $\pm 1,96 S D$ : intervalo de confiança de 95\%. Mean (cm): Média da diferença entre as médias.

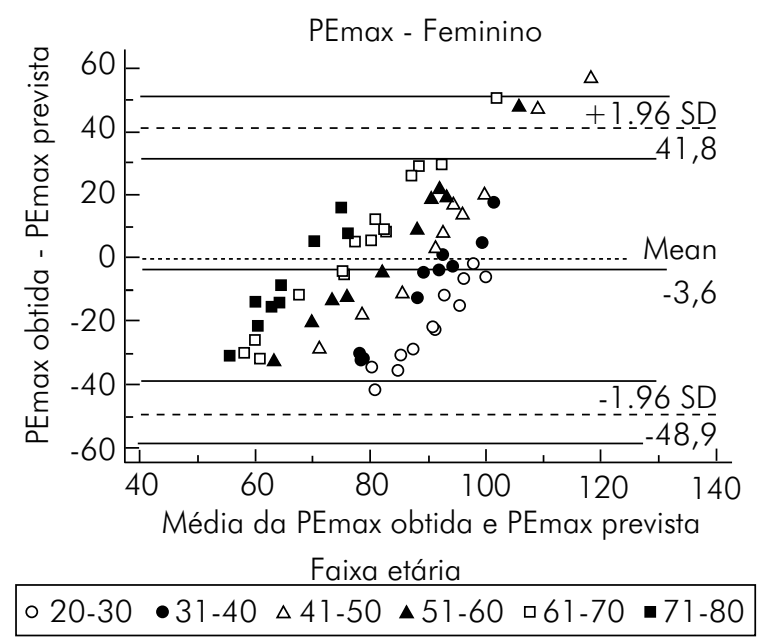




\section{Discussão}

Neste estudo, os valores encontrados de PImáx e PEmáx foram comparados aos valores preditos pelas equaçóes propostas por Neder et al. [3]. Em síntese, esses resultados foram: 1) PImáx obtida tanto nos homens quanto nas mulheres, foram inferiores aos valores preditos, exceto na faixa etária de 61-70 anos nas mulheres e 2) PEmáx obtida tanto para os homens quanto para as mulheres foram inferiores aos valores preditos, exceto na faixa etária de 31-40 anos nos homens.

Em relação aos valores previstos da força muscular respiratória e de seus valores de referência para indivíduos saudáveis, obtidos por meio da utilização de equaçóes de referência em diferentes populaçóes selecionadas, inclusive na população brasileira, acreditamos que é importante estabelecer novas equaçôes que melhor possam predizer os valores de PImáx e PEmáx para a população brasileira, saudável, e dividida em diversas faixas etárias.

Os valores de referência das medidas das pressóes respiratórias, como para muitas variáveis biológicas, devem preferencialmente derivar de uma população geograficamente relacionada e selecionada aleatoriamente na tentativa de melhorar tanto a precisão quanto seu poder preditivo. Sem isto, a interpretação dos resultados dos testes poderia ser impedida e propensa à interpretaçáo errônea [3].

De acordo com nossos resultados, foi possível observar, de maneira geral, que os valores de PImáx e PEmáx obtidos foram menores que os previstos pela equação de Neder $e t a l$. [3] sendo que tanto os valores de PImáx quanto de PEmáx obtidos representaram apenas uma correlação significante para PImáx e uma para PEmáx, assim como para Leal et al. [22] e Parreira et al.[13] cujos resultados também não foram capazes de detectar concordância entre os valores encontrados e preditos na população estudada. Desta forma, isso indica que os resultados demonstrados pela equação do respectivo autor não foram suficientes para predizer os valores de PImáx e PEmáx na população estudada, pois, esses, na maioria das vezes, superestimam os valores obtidos por meio da manovacuometria na população estudada.

Nossos resultados também demonstraram que os homens apresentaram valores de PImáx e PEmáx superiores ao das mulheres em todas as faixas etárias, estando de acordo com os achados de Black e Hyatt [5], Wilson et al. [17], Camelo et al. [20], Bruschi et al. [18], Vincken et al. [23], Enright et al. [24], Berry et al. [15], Harik-Khan et al. [16], Neder et al. [3] e Hautmann et al. [25], Almeida et al. [26]. De acordo com esses autores, os fatores que estariam correlacionados a isto, podem ser as diferenças de dimensões corporais, podendo decorrer da maior força muscular nos homens.

Além disso, apesar de não ter sido explorado neste trabalho a correlação das pressões PImáx e PEmáx com a idade, estudos mostram que a idade influencia significativamente os valores da PImáx e da PEmáx, tanto em homens como em mulheres, sendo que a diminuição dessas duas variáveis ocorrem progressivamente com o avançar de cada década, apresentando assim uma correlação negativa entre a idade e as pressôes respiratórias [3,26,28-30]. Alguns autores justificam esses resultados pela idade estar relacionada às alteraçôes fisiológicas próprias do processo de envelhecimento, como mudanças na composição do tecido pulmonar e da caixa torácica, que acarretam diminuição da massa e da eficiência da musculatura respiratória. Com o processo de senescência, os pulmóes sofrem alteraçôes relacionadas à quantidade e composiçáo dos componentes dos tecidos conjuntivos, promovendo diminuição do recolhimento elástico pulmonar. A caixa torácica sofre progressiva diminuição em sua complacência devido à calcificação da cartilagem das articulaçôes costais e estreitamento dos discos intervertebrais. Alteraçôes na configuração do tórax também ocorrem devido ao processo de osteoporose, que ocasiona microfraturas parciais ou completas das vértebras, levando ao aumento da cifose dorsal e do diâmetro anteroposterior do tórax [27]. Os idosos têm redução na massa muscular do diafragma e da musculatura acessória da respiração, como também têm menor resposta desses músculos a um mesmo nível de estimulação neural, o que justifica ainda mais que pressóes respiratórias máximas apresentam uma relação importante com a idade [3].

Estudos futuros são necessários para avaliar a força muscular respiratória em indivíduos com as mesmas faixas etárias deste estudo, mas em regiôes diferentes, para que possa haver uma comparaçấo dos valores em relação a diferentes regióes do Brasil. Com relação ao tamanho amostral, esta pode ser aumentada, já que esta foi a nossa maior limitação encontrada para conclusão deste trabalho.

\section{Conclusão}

Com base nos resultados obtidos neste estudo conclui-se que os valores previstos propostos por Neder $e t$ al. náo foram suficientemente capazes de predizer os valores de PImáx e PEmáx para os indivíduos da população estudada, pois não houveram correlaçóes positivas significativas entre a maioria dos valores obtidos com os valores previstos pela equação do autor citado. Dessa forma, recomenda-se a elaboração de novos estudos sobre a previsão de valores da PImáx e da PEmáx em indivíduos saudáveis da população brasileira, para que possam servir de parâmetro de normalidade para essa população.

\section{Agradecimento}

CNPq - Processo n. 502727/2007-1.

\section{Referências}

1. Leith DE, Bradley M. Ventilatory muscle strength and endurance training. J Appl Physiol 1976;41:508-16. 
2. Shaffer TH, Wolfson MR, Bhutani VK. Respiratory muscle function assessment and training. Phys Ther 1981;61:795-801.

3. Neder JA, Andreoni S, Lerario MC, Nery LE. Reference values for lung function tests: II. Maximal respiratory pressures and voluntary ventilation. Braz J Med Biol Res 1999;32(6):719-27.

4. Mcconnell AK, Copestake AJ. Maximum static respiratory pressures in healthy elderly men and women: issues of reproducibility and interpretation. Respiration 1999;66:251-8.

5. Black LF, Hyatt RE. Maximal respiratory pressures: normal values and relationship to age and sex. Am Rev Res Dis 1969;99:696-702.

6. Morales P, Sanchis J, Cordero PJ, Díez JL. Maximal static respiratory pressures: reference values for adults. Am Rev Res Dis 1990;141:A-717.

7. Costa D, Jamami M, Valle PHC, Fraianelli VF, Pires VA, Oishi J. Desenvolvimento de um equipamento para treinar a respiração nasal em pacientes com respiração bucal - Nasomanômetro. Anais do VIII Simpósio Internacional de Fisioterapia Respiratória; 1996. p.149.

8. Costa D, Jamami M, Fernandes PM, Oishi J, Thomaz EA, Mellaci VB. Efeito do treinamento da força muscular respiratória em indivíduos sadios. Anais da XII Reunião Anual da Federação de Sociedades de Biologia Experimental 1997; p.342.

9. Costa D. Fisioterapia respiratória básica. São Paulo: Atheneu; 1999.

10. Mangelsdorff G, Borzone T, Leiva G, Martínez S, Lisboa B. Potencia de los músculos inspiratorios en insufiencia cardíaca crónica y en enfermedad pulmonar obtructiva crónica. Rev Méd Chile 2001;129:51-59.

11. Costa D, Sampaio LMM, Lorenzzo VAP, Jamami M, Damaso AR. Avaliação da força muscular respiratória e amplitudes torácicas e abdominais após a RFR em indivíduos obesos. Rev Latinoam Enfermagem 2003;11:156-160.

12. DeTroyer A, Kirkwood PA, Wilson TA. Respiratory action of the intercostal muscles. Physiol Rev 2005;85(2):717-56.

13. Parreira VF, França DC, Zampa CC, Fonseca MM, Tomich GM, Britto RR. Pressóes respiratórias máximas: valores encontrados e preditos em indivíduos saudáveis. Rev Bras Fisioter 2007;11:361-8.

14. Enright PL, Kronmal RA, Manolio TA, Schenker MB, Hyatt RE. Respiratory muscle strength in the elderly. Correlates and reference values. Cardiovascular health study research group. Am J Respir Crit Care Med 1994;149:430-438.

15. Berry JK, Vitalo CA, Larson JL, Patel M, Kim MJ. Respiratory muscle strength in older adults. Nurs Res 1996;45:154-159.

16. Harik-Khan RI, Wise RA, Fozard JL. Determinants of maximal inspiratory pressure. The Baltimore longitudinal study of aging. Am J Respir Crit Care Med 1998;158(5):1459-64.
17. Wilson SH, Cooke NT, Edwards RH, Spiro SG. Predicted normal values for maximal respiratory pressures in caucasian adults and children. Thorax 1984;39:535-8.

18. Bruschi C, Cerveri I, Soia MC, Fanfulla F, Fiorentini M, Casali L, et. al. References values of maximal respiratory mouth pressures: a population-based study. Am Rev Respir Dis 1992;146:790-93.

19. Johan A, Chan CC, Chia HP, Chan O Y, Wang YT. Maximal respiratory pressures in adult Chinese, Malays and Indians. Eur Respir J 1997;10:2825-28.

20. Camelo JS, Terra Filho J, Manço JC. Pressões respiratórias máximas em adultos normais. J Pneumol 1985;11:181-4.

21. Souza RB. Pressões respiratórias estáticas máximas. J Pneumol 2002;28:155-64.

22. Leal AH, Hamasaki TA, Jamami M, Lorenzo VAPD, Pessoa BV. Comparação entre valores de força muscular respiratória medidos e previstos por diferentes equaçóes. Fisioter Pesqui 2007; 14:25-30.

23. Vincken W, Guezzo HE, Cosio MG. Maximal static respiratory pressures in adults: normal values and their relationship to determinants of respiratory function. Bull Eur Physiopathol Respir 1987;23:435-9.

24. Enright PL, Adams AB, Boyle PJR, Sherrill DL. Spirometry and maximal respiratory pressure references from healthy Minnesota 65- to 85-year-old women and men. Chest 1995;108:663-69.

25. Hautmann H, Hefele S, Schotten K, Huber RM. Maximal inspiratory mouth pressures (PImáx) in healthy subjects - what is the lower limit of normal?. Respir Med 2000;94:689-93.

26. Almeida IP, Bertucci NR, Lima VP. Variaçóes da pressão inspiratória máxima e pressão expiratória máxima a partir da capacidade residual funcional ou da capacidade pulmonar total e volume residual em indivíduos normais. O Mundo da Saúde 2008;32:176-82.

27. Simóes RP, Auad MA, Dionísio J, Mazzonetto M. Influence of age and sex on respiratory muscle strength. Fisioter Pesqui 2007; 14:36-41.

28. Carvalho AR, Butzge DM, Bianchini LC, Rocco PF, Rodrigo ACA, Maso GCD, et al. Influência de técnicas manuais na capacidade funcional e mobilidade do gradil costal de idosos. Fit Perf J 2008; 7(5):338-44.

29. Vasconcellos JAC, Britto RR, Parreira VF, Cury A C, Ramiro SM. Respiratory pressures and functional capacity in asymptomatic elderly. Fisioter Mov 2007; 20(3): 93-100.

30. Simóes RP, Auad MA, Dionísio J, Mazzonetto M. Análise comparativa da força muscular respiratória entre idosas institucionalizadas e não institucionalizadas. Fisioter Bras 2006;7(3):191-6. 